\title{
Computer model of a fast toroidal plasma compression, with application to the topolotron
}

\author{
H. Mark Nelson \\ Keith H. Brown \\ Charles A. hart
}

Follow this and additional works at: https://scholarsarchive.byu.edu/facpub

Part of the Astrophysics and Astronomy Commons, and the Physics Commons

\section{Original Publication Citation}

Nelson, Mark H., Keith H. Brown, and Charles A. Hart."Computer model of a fast toroidal plasma compression, with application to the topolotron." Physics of Fluids 19 (1976): 181-1819.

\section{BYU ScholarsArchive Citation}

Nelson, H. Mark; Brown, Keith H.; and hart, Charles A., "Computer model of a fast toroidal plasma compression, with application to the topolotron" (1976). Faculty Publications. 774.

https://scholarsarchive.byu.edu/facpub/774 


\title{
Computer model of a fast toroidal plasma compression, with application to the topolotron
}

\author{
H. Mark Nelson, Keith H. Brown," and Charles A. Hart \\ Brigham Young University, Provo, Utah 84602 \\ (Received 21 November 1975; final manuscript received 28 May 1976)
}

\begin{abstract}
The method is developed for a computer sequence which models a fast toroidal theta or screw pinch for a highly conducting axially symmetric plasma. The computer sequence takes into account the selfinductances of the plasma and the external conductors which drive the compression as well as the mutual inductance which electromagnetically couples the two. The computer sequence is divided into three phases a snowplow compression phase, an adiabatic compression phase, and a crowbarred circuit phase. The computer sequence is applied to a topolotron and an example is given of a magnetohydrodynamic equilibrium geometry for which the plasma surface possesses an asymptotic magnetic field with limit cycles.
\end{abstract}

\section{INTRODUCTION}

The topolotron is a toroidal magnetic plasma confinement device with a noncircular cross section. ${ }^{1}$ It is distinguished from conventional toroidal magnetic plasma confinement devices by the magnetic field configuration on the plasma surface. That configuration is neither closed nor ergodic as is conventional, but rather every field line asymptotically approaches a limit cycle, i. e., an isolated closed field line (see Fig. 1). This asymptotic magnetic field configuration possesses a theoretically advantageous property which is best understood in the context of topology. The topological term "structural stability" is applied to a property which manifests itself physically through the behavior of the magnetic field under small perturbations, viz., the topology of the magnetic field configuration does not change. For example, under a small perturbation, the magnetic field configuration would not change from asymptotic to closed. Conventional toroidal magnetic field configurations do not posses this property, however, and are subject to a change in their topologies under small perturbations, e.g., from ergodic to closed, possibly with a corresponding tendency toward those magnetohydrodynamics instabilities associated with geometrical resonance, e.g., the breaking up into islands of the magnetic structure.

The physical realizability of an asymptotic magnetic field configuration has been studied previously ${ }^{2}$ from the point of view of finding the proper boundary currents required to create a given magnetic field configuration which is known to be asymptotic. That method suffers a serious defect in that the approach to equilibrium is not included. Consequently, the physical requirements of producing the equilibrium are not known. Furthermore, the external currents computed to exist in the presence of those prescribed equilibria which were treated by that method were impractical to produce experimentally, inasmuch as they required precisely programmed surface currents and conductor shapes which were not easily machined. This paper, by contrast, discusses a computer sequence which simulates the compression of a high beta plasma under the influence of given external currents in the sharp boundary model. This compression can be made to ultimately end in equilibrium of the plasma (meaning constant pressure surface). The plasma equilibrium geometry is then examined for the desired asymptotic magnetic field configuration. It turns out that a wide variety of simple external current configurations give rise to plasma equilibria with asymptotic magnetic field configurations, thus circumventing the need for the highly specialized boundary current and conductor shapes derived from given magnetic fields. The motivation for developing the computer compression sequence was to aid in the design of an apparatus to generate an asymptotic magnetic field configuration on a toroidal plasma surface, i. e., to aid in the design of a topolotron. However, the computer sequence is not restricted in its application to the topolotron, but rather it can be applied to any axially symmetric toroidal device for high-beta plasmas.

The simulation of a plasma compression is modeled after an experimentally observed time sequence as follows: Consider a fast straight theta pinch of a high beta plasma. McKenna ${ }^{3}$ has shown that a magnetic field

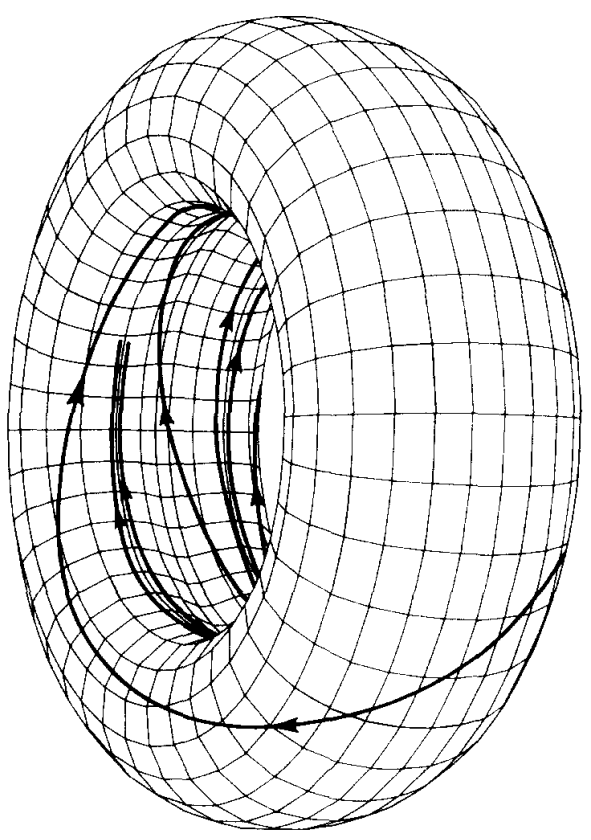

FIG. 1. A torus showing asymptotic field lines on its surface approaching one limit cycle and diverging from another. 
trapped in the plasma (a residual field left from preionization) varies in time as shown in Fig. 2. This graph gives an indication of internal pressure (within the plasma column) during the compression sequence, because the magnetic field lines are "frozen" into the conducting plasma. The gas pressure at the axis of the cylinder appears to remain constant until the plasma, which is being swept up by the imploding magnetic piston, reaches that axis, whereupon there is a rapid increase in the pressure there. Following that rapid rise is a slower increase due to the increasing magnetic pressure on the plasma surface caused by the still-increasing external currents which drive the compression. The time sequence for the simulation is idealized from these observed events. Figure 3 shows the internal pressure of a plasma compressed according to the idealized time sequence. The simulation is meant to model the gross features of a physical plasma. As such it has phenomenological features, rather than purely theoretical ones. It does, however, include the complicated, electromagnetic coupling between a conducting plasma and externally applied currents, not only as it affects the plasma, but also as it affects the distribution of external current, and it represents a practicable way to choose design parameters for an experimental plasma compression. ${ }^{4}$

The first stage of the sequence is a simple snowplow compression $^{5}$ in which the driving currents in external coils together with the induced currents in a perfectly conducting plasma produce a magnetic pressure which compresses the plasma surface rapidly inward and sweeps the interior plasma along with it. The dynamics of the compression are classical, essentially Newton's law: $F=d(m v) / d t$ applied to small sections of the boundary.

The snowplow part of the compression is allowed to continue without a retarding back pressure until the work done by the magnetic driving force has increased to the point where the gas pressure of the plasma computed as will be described is greater than or equal to the average magnetic pressure on the plasma surface. At this time in the sequence, the computer program stops the snowplow sequence and introduces the computed internal plasma pressure as a back pressure which diminishes the effective driving force on the plas-

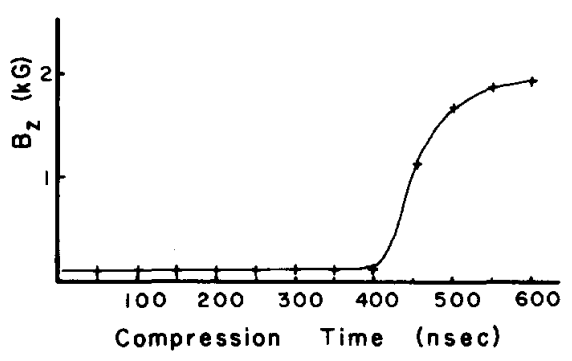

FIG. 2. A graph showing the magnetic field strength on the axis of a straight theta pinch during a fast compression of a high beta plasma. The abscissa is time elapsed since the compression was initiated. Experimental data points are indicated by crosses.

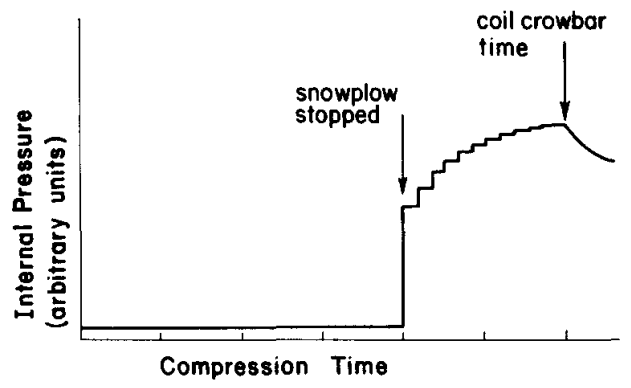

FIG. 3. A schematic drawing showing internal pressure of a plasma compressed according to a time sequence idealized from the experimental time sequence of Fig. 2. The abscissa is time elapsed since the compression was initiated.

ma surface. Moreover, at this point in the sequence, the adiabatic ideal gas relationship is introduced: $P V^{\gamma}$ $=$ const, where $\gamma$ is the ratio of heat capacity at constant pressure to that at constant volume, so that, with subsequent increments of time, this equation governs the relationship between pressure and volume. The volume is changed, in these subsequent computer sequences, by moving each section of the plasma boundary in a direction perpendicular to the boundary and by an amount proportional to the difference between the magnetic pressure computed for that section and the internal gas pressure. If the external currents lie within certain (fairly nonrestrictive) limits, the procedure moves the boundary in such a way that equilibrium (i. e., constant magnetic pressure on the boundary equal to the internal gas pressure) is approached. This process is iterated until the circuit crowbarring time, which is chosen to be that time when any one of the external currents changes sign, about one-quarter period of the oscillatory discharge of the external capacitor bank through the coil inductors. This repeated equilibrium-seeking sequence corresponds to the stepped portion of the graph in Fig. 3. At the circuit crowbar time, the circuit equations are altered to represent the shorted coils (crowbar condition). At this time a circuit resistance is introduced. The equilibrium-seeking process is continued as long as would be physically interesting, as the currents in the coils gradually decay due to the circuit resistance.

The method of computing magnetic pressure will be discussed in Sec. II. Detailed descriptions of the snowplow, adiabatic compression, and crowbar portions of the computer sequence will be given in Secs. III, IV, and $V$, respectively. Section VI will give an example of the application of the computer sequence to the topolotron.

\section{COMPUTATION OF MAGNETIC PRESSURE}

The basic equations governing equilibrium in magnetofluid dynamics are

$$
\begin{aligned}
& \nabla \cdot \mathrm{B}=0, \\
& \nabla \times \mathrm{B}=\mu \mathrm{j}, \\
& \mathrm{j} \times \mathrm{B}=\nabla P,
\end{aligned}
$$

where the first two equations are simply time independent Maxwell's equations in mks units, and the third 
equation expresses the balance between magnetic pressure and fluid pressure, $P$. These equations can be combined to give the result: $P+B^{2} / 2 \mu$ is constant across the plasma boundary. For the sharp-boundary perfect-conductor model, moreover, the magnetic field within the conductor (plasma) vanishes so that the internal gas pressure balances the external magnetic pressure. This result from magnetofluid dynamics is used in the computer sequence to calculate the plasma equilibrium. ${ }^{6}$

In the computer model discussed here, the high-beta toroidal plasma is regarded as a perfect conductor. Currents are induced in the surface when the driving currents in external coils are turned on. Currents arise from capacitor banks discharging through the external coils. There are three external coils as shown in Fig. 4: (1) a poloidally wound coil (hereafter called the $\theta$-coil), (2) a toroidally wound outer coil (called the $\mathrm{C}$ coil), and (3) a toriodally wound inner coil (called the I coil). The currents in the external coils and in the plasma surface are governed by the coupled effects of the discharging capacitors and the inductance of the circuits. Faraday's law and the loop theorem require that the time rate of change of the flux linking a conductor be equal to the emf applied to it. If there is no applied emf, as in the case of the plasma, then the flux linking the conductor remains constant:

$$
\dot{\Phi}=\frac{d}{d t} \int_{s} \mathrm{~B} \cdot \hat{n} d S=0 .
$$

For the case of the external conductors:

$$
\dot{\Phi}=\frac{d}{d t} \int_{s} \mathrm{~B} \cdot \hat{n} d S=-\mathcal{E}
$$

where $\mathcal{E}$ is the applied emf due to the capacitance and any other source of emf which may be present. Note that the flux, $\Phi_{i}$, linking a given conducting circuit may

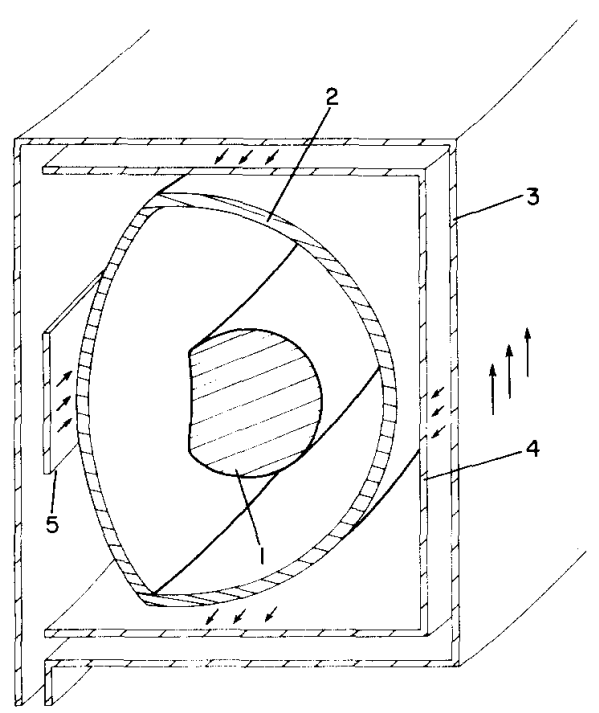

FIG. 4. A view of (1) the poloidal cross section of a plasma with two limit cycles. Also shown are (2) the vacuum envelope, (3) the $\theta$ coil, (4) the $\mathrm{C}$ coil, and (5) the I coil. The directions of the currents in the coils are indicated with arrows. be written in terms of the inductances and currents as follows:

$$
\Phi_{i}=\int_{s_{i}} \mathbf{B} \cdot \hat{n} d S=\sum_{j} L_{i j} J_{j}
$$

where $L_{i j}$ is the inductance between the $i$ th and $j$ th circuits and $J_{j}$ is the current in the $j$ th circuit. These flux conditions based on Faraday's law are, in turn, the basis upon which the currents in the various conductors may be calculated, and subsequently, the pressure which drives the plasma compression is obtained. The fact that the $C$ coil and I coil are orthogonal to the $\theta$ coil allows us to treat separately the problems of calculating the current induced in the plasma by driving currents in these orthogonal coils. In applying the computer sequence to the topolotron, the I and $\mathrm{C}$ coils will carry oppositely directed currents. The computer sequence is not restricted to these $I$ and $C$ coil shapes, or current directions, but applies to any axially symmetric arrangement of orthogonal conductors.

\section{A. Pressure due to the $\theta$ coil}

The flux condition for the toroidal flux linking the plasma and the $\theta$ coil may be written:

$$
\begin{aligned}
& \dot{\Phi}_{D}=\frac{d}{d t} \int_{s_{p}} \mathrm{~B}_{t} \cdot \hat{n} d s=0 \\
& \dot{\Phi}_{\theta}=\frac{d}{d t} \int_{s_{\theta}} \mathrm{B}_{t} \cdot \hat{n} d S=-\mathcal{E}=-Q_{1} / C-L_{e} \ddot{Q}_{1},
\end{aligned}
$$

where the emf applied to the $\theta$ coil consists of $Q_{1} / C$ due to the capacitance and $L_{e} \ddot{Q}_{1}$ due to lead inductance. The charge on the capacitor is $Q_{1}$, and the superior dots indicate the time derivatives. From the first of these flux conditions, $\Phi_{p}$ is constant in time, and we shall investigate the case where it vanishes. Both the $\theta$ coil current, $\dot{Q}_{1}$, and the induced plasma current, $\dot{Q}_{2}$, are poloidally directed and the magnetic field produced by them is

$$
\mathrm{B}_{t}=\left(\mu_{0} \dot{Q}_{1} / 2 \pi r\right) \hat{e}_{\phi}
$$

in the space between the $\theta$ coil and the plasma, or

$$
\mathrm{B}_{t}=\left[\mu_{0}\left(\dot{Q}_{1}+\dot{Q}_{2}\right) / 2 \pi r\right] \hat{e}_{\phi}
$$

within the plasma. No magnetic field arises in the region outside a torus for poloidal surface currents. Here, $\mu_{0}$ is the permeability of free space $\left(\mu_{0}=4 \pi \times 10^{-7}\right.$ in mks units), $r$ is the distance from the axis of the torus, and $\hat{e}_{\phi}$ is a unit vector in the toroidal direction. The vanishing of $\Phi_{p}$ implies that $B_{t}$ vanishes, inside the perfectly conducting plasma. Consequently, the flux linking the $\theta$ coil may be written

$$
\Phi_{\theta}=\int_{s_{\theta}}\left(\mu_{0} / 2 \pi r\right) \dot{Q}_{1} d S-\int_{s_{p}}\left(\mu_{0} / 2 \pi r\right) \dot{Q}_{1} d S
$$

The time derivative of a surface integral for which the surface changes in time, can be written as

$$
\frac{d}{d t} \int_{s} \mathrm{~B} \cdot \hat{n} d S=\int_{s} \hat{n} \cdot\left[\frac{\partial \mathrm{B}}{\partial t}+\mathrm{v}(\nabla \cdot \mathrm{B})+\nabla \times(\mathrm{B} \times \mathrm{v})\right] d S,
$$

where $\mathrm{v}$ is the velocity of the surface element. For a magnetic field, $\nabla \cdot B=0$, from Maxwell's equation, and by Stokes theorem, the last term in the integral may be 
changed to a line integral around the boundary of the surface

$$
\frac{d}{d t} \int_{s} \mathrm{~B} \cdot \hat{n} d S=\int_{s} \frac{\partial \mathrm{B}}{\partial t} \cdot \hat{n} d S+\int_{c} \mathrm{~B} \times \mathrm{v} \cdot \mathrm{d} l .
$$

Upon applying this result to $\Phi_{\theta}$, one obtains

$$
\dot{\Phi}_{\theta}=\int_{s_{\theta}}\left(\frac{\mu_{0} \ddot{Q}_{1}}{2 \pi r}\right) d S-\int_{s_{p}}\left(\frac{\mu_{0} \ddot{Q}_{1}}{2 \pi r}\right) d S-\int_{c_{p}}\left(\frac{\mu_{0} \dot{Q}_{1} v_{p}}{2 \pi r}\right) d l,
$$

where use has been made of the fact that the $\theta$ coil does not move and it is assumed that $B_{t} \times v_{p}$ is tangential to the boundary of the poloidal cross section of the plasma, i. e., that $v_{p}$ is perpendicular to that boundary. Using these results one may write the flux condition for the $\theta$ coil as follows:

$$
\dddot{Q}_{1}-2 \beta \dot{Q}_{1}+\omega_{0}^{2} Q_{1}=0,
$$

where

$$
\omega_{0}^{2}=\left(C\left(L_{e}+\frac{\mu_{0}}{2 \pi} \int_{s_{\theta}} \frac{d S}{r}-\frac{\mu_{0}}{2 \pi} \int_{s_{p}} \frac{d s}{r}\right)\right)^{-1}
$$

and

$$
\beta=\frac{1}{2} C \omega_{0}^{2}\left[\frac{\mu_{0}}{2 \pi} \int_{c_{p}}\left(\frac{v_{p}}{r}\right) d l\right] .
$$

Because the plasma surface is moving, $\beta$ and $\omega_{0}$ are not constant; however, they are slowly varying in the sense that $\Delta \beta / \beta$ or $\Delta \omega_{0} / \omega_{0}$ remains less than $10^{-2}$ during an iteration. For this reason, one may numerically solve the differential equation by letting $\beta$ and $\omega_{0}$ be fixed at their initial values for a short interval of time; next, solving the resulting equation analytically as will be shown, and then obtaining new values for $\beta$ and $\omega_{0}$ which lead to a new solution, etc. The solution of the differential equation for the $n$th iteration is

$$
\begin{aligned}
Q_{1}\left(t_{n}\right)= & \exp \left(\beta_{n} \Delta t\right)\left[Q_{1}\left(t_{n-1}\right) \cos \left(\omega_{n} \Delta t\right)\right. \\
& +\omega_{n}^{-1}\left(\dot{Q}_{1}\left(t_{n-1}\right)-\beta_{n} Q_{1}\left(t_{n-1}\right)\right] \sin \left(\omega_{n} \Delta t\right),
\end{aligned}
$$

where

$$
\Delta t=t_{n}-t_{n-1}
$$

and

$$
\omega_{n}^{2}=\omega_{0_{n}}^{2}-\beta_{n}^{2} .
$$

The accuracy of this numerical method for solving the differential equation was checked by decreasing the time interval by a factor of five. The corresponding change in $Q_{1}\left(t_{n}\right)$ after sixty iterations was less than $0.02 \%$. The current is similarly given by the expression:

$$
\begin{aligned}
\dot{Q}_{1}\left(t_{n}\right)= & \exp \left(\beta_{n} \Delta t\right)\left\{\operatorname { c o s } ( \omega _ { n } \Delta t ) \left[\dot{Q}_{1}\left(t_{n-1}\right)\right.\right. \\
& \left.\left.-\beta_{n} Q_{1}\left(t_{n-1}\right)\right]-\omega_{n} Q_{1}\left(t_{n-1}\right) \sin \left(\omega_{n} \Delta t\right)\right\}+\beta_{n} Q_{1}\left(t_{n}\right) .
\end{aligned}
$$

Once the current, $\dot{Q}_{1}$, is obtained, the toroidal component of magnetic field can be computed from the formula: $B_{t}=\mu_{0} \dot{Q}_{1} / 2 \pi r$, and the contribution of this field component to the magnetic pressure at the plasma surface is given by the magnetohydrodynamic equation $P_{t}=B_{t}^{2} / 2 \mu_{0}$.

\section{B. The pressure due to the $I$ and $C$ coils}

The poloidal flux condition for the I coil, the $\mathrm{C}$ coil, and the plasma are, respectively:

$$
\begin{aligned}
& \dot{\Phi}_{I}=-L_{I} \ddot{Q}_{I}-Q_{I} / C_{I}, \\
& \dot{\Phi}_{C}=-L_{C} \ddot{Q}_{C}-Q_{C} / C_{C}, \\
& \dot{\Phi}=0 .
\end{aligned}
$$

The poloidal flux linking each of these conductors may be conceptually decomposed into three contributions, namely, those originating from the toroidal currents in these conductors. This decomposition is simply an application of the principle of superposition for magneticfields. Each of these flux contributions may be further decomposed by imagining the conducting surface as toroidally segmented. Because $\hat{n} \cdot \mathrm{B}=0$ for a perfectly conducting surface (i.e., the magnetic field is tangential to the surface), each surface segment of a given conductor is linked by the same flux:

$$
\begin{aligned}
& \Phi_{I}=\sum_{j=1}^{n_{1}} \Phi_{i j}^{I I}+\sum_{j=n_{1+1}}^{n_{1}+n_{2}} \Phi_{i j}^{I C}+\sum_{j=n_{1}+n_{2}+1}^{n_{1}+n_{2}+n_{3}} \Phi_{i j}^{I P}, i=1, \ldots, n_{1} \\
& \Phi_{C}=\sum_{j=1}^{n_{1}} \Phi_{i j}^{C I}+\sum_{j=n_{1}+1}^{n_{1}+n_{2}} \Phi_{i j}^{C C}+\sum_{j=n_{1}+n_{2}+1}^{n_{1}+n_{2}+n_{3}} \Phi_{i j}^{C P}, \\
& i=n_{1}+1, \ldots, n_{1}+n_{2} \\
& \Phi_{P}=\sum_{j=1}^{n_{1}} \Phi_{i j}^{P I}+\sum_{j=n_{1}+1}^{n_{1}+n_{2}} \Phi_{i j}^{P C}+\sum_{j=n_{1}+n_{2}+1}^{n_{1}+n_{2}^{+n_{3}}} \Phi_{i j}^{P P}, \\
& i=n_{1}+n_{2}+1, \ldots, n_{1}+n_{2}+n_{3}
\end{aligned}
$$

where the first and second superscripts indicate the conductor linked by the flux and the conductor which is the source of the flux, respectively, and where the first and second subscripts indicate particular surface segments of these two conductors, respectively. There are $n_{1}$, I-coil segments, $n_{2}, \mathrm{C}$-coil segments, and $n_{3}$, plasma surface segments. The motivation for treating the conducting surfaces as segmented, is that the toroidal current density in these conductors is thereby rendered accessible. ${ }^{7}$ The inductance, $L_{i j}$ of a circuit is defined in terms of the flux, $\Phi_{i j}$, linking that circuit and the current, $J_{j}$, which is the source of the flux as follows:

$$
\Phi_{i j}=L_{i j} J_{j} \cdot
$$

The foregoing flux conditions provide a set of differential equations from which the currents in the surface segments may be computed. Those currents divided by the length of the surface segment give the average surface current density in the segment.

The differential equations given by the flux conditions are the following, in matrix notation:

$$
\begin{aligned}
& -\mathbf{E}^{I}=\frac{d}{d t}\left[\mathrm{~L}^{I I} \mathrm{~J}^{I}+\mathrm{L}^{I C} \mathrm{~J}^{C}+\mathrm{L}^{I P} \mathrm{~J}^{P}\right], \\
& -\mathrm{E}^{C}=\frac{d}{d t}\left[\mathrm{~L}^{C I} \mathrm{~J}^{I}+\mathrm{L}^{C C} \mathrm{~J}^{C}+\mathrm{L}^{C P} \mathrm{~J}^{P}\right], \\
& 0=\frac{d}{d t}\left[\mathrm{~L}^{P I} \mathrm{~J}^{I}+\mathrm{L}^{P C} \mathrm{~J}^{C}+\mathrm{L}^{P P} \mathrm{~J}^{P}\right],
\end{aligned}
$$

where $\mathbf{E}^{I}$ and $\mathbf{E}^{c}$ are column matrices having $n_{1}$ identical elements $L_{I} \ddot{Q}_{I}+Q_{I} / C_{I}$ and $n_{2}$ identical elements $L_{C} \ddot{Q}_{C}$ $+Q_{C} / C_{C}$, respectively. The elements of the column 
matrices, $\mathrm{J}^{I}, \mathrm{~J}^{C}$ or $\mathrm{J}^{P}$ are the toroidal currents in the $n_{1}, n_{2}$, or $n_{3}$ surface segments of the I coil, the $\mathrm{C}$ coil, or the plasma surface, respectively. The nine inductance matrices, $L^{A B}$, are labeled by the conductors being coupled, and their dimensionalities are determined by the number of surface segments treated in each conductor.

It is convenient to further combine these matrices as follows:

$$
\begin{aligned}
& \mathrm{E}^{E} \equiv\left[\begin{array}{l}
\mathrm{E}^{I} \\
\mathrm{E}^{c}
\end{array}\right], \quad \mathrm{J}^{E} \equiv\left[\begin{array}{l}
\mathrm{J}^{I} \\
\mathrm{~J}^{C}
\end{array}\right], \quad \mathrm{L}^{E E} \equiv\left[\begin{array}{l}
\mathrm{L}^{I I} \mathrm{~L}^{I C} \\
\mathrm{~L}^{C I} \mathrm{~L}^{C C}
\end{array}\right], \\
& \mathrm{L}^{E P} \equiv\left[\begin{array}{l}
\mathrm{L}^{I P} \\
\mathrm{~L}^{C P}
\end{array}\right], \quad \mathrm{L}^{P E} \equiv\left[\mathrm{L}^{P I}, \mathrm{~L}^{P C}\right] .
\end{aligned}
$$

where the superscript $E$ refers to the external conductors and currents, i. e., external to the plasma. With this notation, the matrix equations become

$$
\begin{aligned}
& -\mathrm{E}^{E}=\frac{d}{d t}\left[\mathrm{~L}^{E E} \mathrm{~J}^{E}+\mathrm{L}^{E P} \mathrm{~J}^{P}\right] \\
& 0=\frac{d}{d t}\left[\mathrm{~L}^{P E} \mathrm{~J}^{E}+\mathrm{L}^{P P} \mathrm{~J}^{P}\right] .
\end{aligned}
$$

The second of these equations may be integrated, and we will treat the case where the integration constant matrix vanishes. This case corresponds to the physical situation where no poloidal flux links the plasma. For this case one may solve for the plasma current matrix, if $\mathrm{L}^{P P}$ has an inverse

$$
\mathrm{J}^{P}=-\left(\mathrm{L}^{P P}\right)^{-1} \mathrm{~L}^{P E} \mathrm{~J}^{E} .
$$

Using this expression in the first matrix equation, one gets

$$
-\mathrm{E}^{E}=\frac{d}{d t}\left[\left(\mathrm{~L}^{E E}-\mathrm{L}^{E P}\left(\mathrm{~L}^{P P}\right)^{-1} \mathrm{~L}^{P E}\right) \mathrm{J}^{E}\right] \equiv \frac{d}{d t}\left(\mathbf{K} \mathrm{J}^{E}\right),
$$

where $\mathbf{K}$ is defined through this equation. This equation may now be integrated and solved for $\mathbf{J}^{E}$ when $\mathbf{K}$ has an inverse

$$
\mathrm{J}^{E}=-\mathrm{K}^{-1} \int \mathrm{E}^{E} d t
$$

where $\int \mathrm{E}^{E} d t$ is a column matrix having $L_{I} \dot{Q}_{I}+C_{I}^{-1} \int Q_{I} d t$ for its first $n_{1}$ elements and $L_{c} \dot{Q}_{C}+C_{C}^{-1} \int Q_{C} d t$ for its next $n_{2}$ elements. Next consider the equations relating charge on the capacitors and current in the circuits:

$$
\dot{Q}_{I}=\sum_{i=1}^{n_{1}} J_{i}^{E}, \quad \dot{Q}_{C}=\sum_{i=n_{1}+1}^{n_{1}+n_{2}} J_{i}^{E} .
$$

One may write

$$
\begin{aligned}
& \dot{Q}_{I}=-\sum_{i=1}^{n_{1}} \sum_{j=1}^{n_{1}}\left(\mathrm{~K}^{-1}\right)_{i j}\left(C_{I}^{-1} \int Q_{I} d t+L_{I} \dot{Q}_{I}\right) \\
& -\sum_{i=1}^{n_{1}} \sum_{i=n^{+1}}^{n_{1}{ }^{+n_{2}}}\left(\mathbf{K}^{-1}\right)_{i j}\left(C_{C}^{-1} \int Q_{C} d t+L_{C} \dot{Q}_{C}\right) \\
& \dot{Q}_{C}=\sum_{i=n^{+1}}^{n_{1}+n_{2}} \sum_{j=1}^{n_{1}}\left(K^{-1}\right)_{i j}\left(C_{I}^{-1} \int Q_{I} d t+L_{I} \dot{Q}_{I}\right) \\
& -\sum_{i=n_{1}^{+1}}^{n_{1}+n_{2}} \sum_{j=n_{1}^{+1}}^{n_{1}+n_{2}}\left(K^{-1}\right)_{i j}\left(C_{C}^{-1} \int Q_{C} d t+L_{C} \dot{Q}_{C}\right)
\end{aligned}
$$

or in matrix form

$$
(1+\mathrm{ML}) \dot{\mathrm{Q}}+\mathrm{MC}^{-1} \int \mathrm{Q} d t=0,
$$

where

$$
\mathbf{Q}=\left[\begin{array}{l}
Q_{I} \\
Q_{C}
\end{array}\right], \quad \mathbf{L}=\left[\begin{array}{l}
L_{I} O \\
O L_{C}
\end{array}\right], \quad \mathbf{C}=\left[\begin{array}{l}
C_{I} O \\
O C_{C}
\end{array}\right]
$$

and

$$
\mathbf{M}=\left[\begin{array}{ll}
\sum_{i=1}^{n_{1}} \sum_{j=1}^{n_{1}}\left(\mathbf{K}^{-1}\right)_{i j} & \sum_{i=1}^{n_{1}} \sum_{j=n_{1}+1}^{n_{1}+n_{2}}\left(K^{-1}\right)_{i j} \\
\sum_{i=n_{1}+1}^{n_{1}+n_{2}} \sum_{j=1}^{n_{1}}\left(K^{-1}\right)_{i j} & \sum_{i=n_{1}+1}^{n_{1}+n_{2}} \sum_{j=n_{1}+1}^{n_{1}+n_{2}}\left(K^{-1}\right)_{i j}
\end{array}\right]
$$

One may write this as

$$
\grave{\mathbf{Q}}+\omega_{0}^{2} \int \mathbf{Q} d t=0,
$$

where

$$
\omega_{0}^{2}=(1+\mathrm{ML})^{-1} \mathbf{M C}^{-1} \text {. }
$$

Upon differentiating, one gets the equation

$$
\ddot{\mathbf{Q}}-\left[\frac{d}{d t}\left(\omega_{0}^{2}\right)\right]\left(\omega_{0}^{2}\right)^{-1} \dot{\mathbf{Q}}+\omega_{0}^{2} \mathbf{Q}=0
$$

which has the solution

$$
\mathbf{Q}(t)=\exp (\beta t)[\cos (\omega t) \mathbf{A}+\sin (\omega t) \mathbf{B}],
$$

where

$$
\beta=\frac{1}{2}\left[\frac{d}{d t}\left(\omega_{0}^{2}\right)\right]\left(\omega_{0}^{2}\right)^{-1}, \quad \omega^{2}=\omega_{0}^{2}-\beta^{2},
$$

and where $\mathbf{A}$ and $\mathbf{B}$ are (column) matrices of integration. This form for the solution involves the condition that $\beta$ commute with $\omega_{0}^{2}$. In practice, the commutator was found to be of the order of 0.01 times the product of the matrices. The sinusoidal and exponential functions of matrices are to be understood in the sense of matrix power series. In terms of the matrices $\mathbf{Q}\left(t_{0}\right)$ and $\dot{\mathbf{Q}}\left(t_{0}\right)$ evaluated at $t=t_{0}$, the charge and current matrices $\mathbf{Q}(t)$ and $\dot{\mathbf{Q}}(t)$ at any time $t$ are given by

$$
\begin{aligned}
\mathbf{Q}(t)= & \exp \left[\beta\left(t-t_{0}\right)\right]\left\{\cos \left[\omega\left(t-t_{0}\right)\right] \mathbf{Q}\left(t_{0}\right)\right. \\
& \left.+\omega^{-1} \sin \left[\omega\left(t-t_{0}\right)\right]\left[\dot{\mathbf{Q}}\left(t_{0}\right)-\beta \mathbf{Q}\left(t_{0}\right)\right]\right\} \\
\dot{\mathbf{Q}}(t)= & \exp \left[\beta\left(t-t_{0}\right)\right]\left\{\cos \left[\omega\left(t-t_{0}\right)\right]\left[\dot{\mathbf{Q}}\left(t_{0}\right)-\beta \mathbf{Q}\left(t_{0}\right)\right]\right. \\
& \left.-\omega \sin \left[\omega\left(t-t_{0}\right)\right\} \mathbf{Q}\left(t_{0}\right)\right\}+\beta \mathbf{Q}(t) .
\end{aligned}
$$

The solution of the matrix equation in this form is especially adapted to computer computations in which $\mathbf{Q}(t)$ and $\dot{\mathbf{Q}}(t)$ are given in terms of their values in a previous iteration.

Next, use the equation

$$
\mathbf{J}^{E}=\mathbf{K}^{-1} \int \mathbf{E}^{E} d t
$$

In component form

$$
\begin{aligned}
J_{i}^{E}= & \sum_{j=1}^{n_{1}}\left(\mathbf{K}^{-1}\right)_{i j}\left(C_{I}^{-1} \int Q_{I} d t+L_{I} \dot{Q}_{I}\right) \\
& +\sum_{j=n_{1}+1}^{n_{1}+n_{2}}\left(\mathbf{K}^{-1}\right)_{i j}\left(C_{C}^{-1} \int Q_{C} d t+L_{C} \dot{Q}_{C}\right)
\end{aligned}
$$


From the equation $\dot{\mathbf{Q}}+\omega_{0}^{2} \int \mathbf{Q} d t=0$, one can see that $\int Q d t=\left[\begin{array}{l}\int Q_{I} d t \\ \int Q_{C} d t\end{array}\right]=-\left[\begin{array}{c}\left(\omega_{0}^{2}\right)_{I I}^{-1} \dot{Q}_{I}+\left(\omega_{0}^{2}\right)_{I C}^{-1} \dot{Q}_{C} \\ \left(\omega_{0}^{2}\right)_{C I}^{-1} \dot{Q}_{I}+\left(\omega_{0}^{2}\right)_{c C}^{-1} \dot{Q}_{C}\end{array}\right]$, so that one may write the following expression for $J_{i}^{E}$

$$
\begin{aligned}
J_{i}^{B}= & \sum_{j=1}^{n_{1}}\left(K^{-1}\right)_{i j}\left[\left(L_{I}-C_{I}^{-1}\left(\omega_{0}^{2}\right)_{I I}^{-1}\right)_{Q_{I}}-C_{I}^{-1}\left(\omega_{0}^{2}\right)_{I C}^{-1} \dot{Q}_{C}\right] \\
& +\sum_{j=n_{1}+1}^{n_{1}^{+n_{2}}}\left(K^{-1}\right)_{i j}\left[\left(L_{C}-C_{C}^{-1}\left(\omega_{0}^{2}\right)_{C C}^{-1}\right)_{Q_{C}}-C_{C}^{-1}\left(\omega_{0}^{2}\right)_{C I}^{-1} \dot{Q}_{I}\right] .
\end{aligned}
$$

The plasma currents $J_{j}^{P}$ are obtained from the matrix equation

$$
J^{P}=-\left(L^{P P}\right)^{-1} L^{P B} J^{E} .
$$

Once the currents $J_{j}^{P}$ are obtained, the contribution to the magnetic pressure of the poloidal component of magnetic field, $B_{p}$, is computed as follows: The boundary condition $^{8}$

$$
\hat{n} \times\left(\mathrm{B}_{+}-\mathrm{B}_{-}\right)=\mu \boldsymbol{X}
$$

for the change of tangential component of magnetic field across a surface current is used. For the perfect conductor, $B_{-}=0$ and

$$
\left|\hat{n} \times \mathrm{B}_{+}\right|=B_{P} \text {. }
$$

The average surface current density $|\mathscr{X}|$ at the position of the $j$ th surface segment is $J_{j}^{P} / \Delta l_{j}$, where $\Delta l_{j}$ is the length of plasma boundary which is alloted to the $j$ th segment. Using these relationships, the contribution to the magnetic pressure at the $j$ th segment from this source is given by

$$
\left(B_{p}^{2} / 2 \mu_{0}\right)_{j}=\frac{1}{2}\left(J_{j}^{P}\right)^{2} \mu_{0} /\left(\Delta l_{j}\right)^{2} .
$$

We make use of the reflection symmetry in the midplane to reduce the dimensions of the matrices to onehalf their original sizes. The self-inductance $L_{i t}$ of a single turn of thin conducting tape of mean radius $R_{t}$ and width $r_{i}$ is given approximately by Grover. ${ }^{9}$ For $R_{i} \gg r_{i}$, the orientation of the strip is irrelevant. The mutual inductance $L_{i j}$ between two concentric circular filaments is well known. ${ }^{8}$ Expressions for these inductances are shown here in mks units

$$
\begin{aligned}
& L_{i i}=R_{i} \mu_{0}\left[\ln \left(8 R_{i} / r_{i}\right)-\frac{1}{2}\right], \\
& L_{i j}=\left(\mu_{0} R_{i}^{1 / 2} R_{j}^{1 / 2} / k\right)\left[\left(2-k^{2}\right) K(k)-2 E(k)\right], \\
& k^{2}=4 R_{i} R_{j}\left[\left(R_{i}+R_{j}\right)^{2}+\left(z_{i}-z_{j}\right)^{2}\right]^{-1},
\end{aligned}
$$

where $z_{i}-z_{j}$ is the axial separation of the concentric loops and where $K$ and $E$ are complete elliptic integrals of the first and second kind, respectively.

\section{THE SNOWPLOW MODEL}

In the snowplow model of the plasma compression, the magnetic pressure on the surface of the plasma implodes the plasma surface inward, sweeping in the plasma as it moves. To calculate this movement, the poloidal cross section of the plasma surface is considered to be composed of several segments which are identified with the segments used in the calculations of the I and $C$ coil currents. Under the influence of the magnetic pressure, which is computed as described in the previous sections, each segment is assumed to be accelerated in a direction perpendicular to a line reaching between midpoints of the adjacent segments. The equation of motion is $F=d(m v) / d t$, where $F$ is a constant force (during a short time interval), $m$ is the mass of the moving portion of the plasma, and $v$ is its velocity. This procedure is dictated by the fact that the force is not given a priori but rather is dependent upon the movement. The method converges as evidenced by the fact that shorter time increments make negligible difference to the results obtained in the cases tried (see Sec. VI).

Upon integrating, one gets: $F\left(t-t_{0}\right)=M v-m_{0} v_{0}$, where $m_{0}, v_{0}$, and $t_{0}$ refer to the beginning of the time interval. The plasma mass is being swept up according to the relationship: $d m / d t=\rho A v$, where $\rho$ is the (uniform) uncompressed plasma density and $A$, the surface area in this section of plasma surface $\left(A_{j}=\Delta l_{j} 2 \pi r_{j}\right.$, where $r_{j}$ is the average radius of the $j$ th segment). Substituting $v$ from this equation into the previous equation, one gets $(m / p A)(d m / d t)=m_{0} v_{0}+F\left(t-t_{0}\right)$. Upon integrating, one obtains $m=m_{0}\left[1+2 \rho A v_{0}\left(t-t_{0}\right) / m_{0}+F \rho A\left(t-t_{0}\right)^{2} /\right.$ $\left.m^{2}\right]^{1 / 2}$. By using this expression for $m$ in the equation $v=x=\left[m_{0} v_{0}+F\left(t-t_{0}\right)\right] / m$, one arrives at the expression, $x-x_{0}=\left(m-m_{0}\right) / \rho A$. Of course, the force is not constant during the compression because the inductance matrices depend on plasma position and because the capacitors are discharging. Consequently, after a short interval of time, during which the plasma moves a distance $\left(x-x_{0}\right)$, a new force and area are computed, again giving rise to a plasma surface movement, etc. After each movement of the plasma, the midpoints of the surface segments are slightly moved along the surface in order to maintain segments of equal length. The plasma mass associated with each segment was not adjusted after this movement. The reason for shifting the surface segments is that the segments in a convex region of the surface tend to cross over each other during the compression causing computational difficulties. The effect of shifting the surface segments in this way is a slight migration of mass away from highly convex regions of the plasma surface and toward slightly convex or concave regions. The mass, velocity, and position of the $n+1$ st iteration are related to the mass, velocity, and position of the $n$th iteration as

$$
\begin{aligned}
& M_{n+1}=m_{n}\left[1+2 \rho A_{n} v_{n} \Delta t_{n} / m_{n}+F_{n} \rho A_{n}\left(\Delta t_{n}\right)^{2} / m_{n}^{2}\right]^{1 / 2}, \\
& v_{n+1}=\left(m_{n} v_{n}+F_{n} \Delta t_{n}\right) / m_{n+1}, \\
& x_{n+1}=x_{n}+\left(m_{n+1}-m_{n}\right) / \rho A_{n} .
\end{aligned}
$$

The work done by the magnetic driving force after $n$ increments of time is taken to be $\sum_{i=1}^{n}\left(m_{i} v_{i}-m_{i-1} v_{i-1}\right)$ summed over all the sections of the plasma. This work plus the initial internal plasma energy is interpreted to be the internal plasma energy, $E_{n}$, after $n$ increments of time. The internal energy, $E$, of an ideal gas in equilibrium is related to its pressure and volume through the equation $P V=(2 / 3) E$. Consequently, a pressure $P_{n}=(2 / 3) E_{n} / V_{n}$ is computed after each increment of time in the computer sequence, and this simulated gas 
pressure is compared with the average magnetic pressure $\bar{P}$ (mag) on the surface of the plasma. When $P_{n}$ (gas) $>\bar{P}$ (mag), the snowplow portion of the computer sequence is stopped and an adiabatic compression stage is initiated as the currents continue to increase until crowbar time is reached.

The snowplow sequence just described involves some rather severe approximations. First, the collisions of the plasma surface with the particles of the interior plasma are considered to be completely inelastic, whereas the whole range between elastic and inelastic collisions is to be expected. This assumption for the snowplow model implies that the interior plasma pressure remains constant. Second, the velocity components of the surface segments which are tangent to the plasma surface are ignored (or, more precisely, the total velocity of each segment is redirected at each iteration to remain perpendicular to the surface). Third, the segment lengths are adjusted at each iteration, although their masses are not.

The neglect of tangential velocity and the adjustment of segment length probably result in errors smaller than those introduced by the snowplow model itself, particularly since the snowplow sequence ends rather early in the compression (see contour 2 of Fig. 5) when most of the plasma surface segments are still being accelerated approximately in the direction of their initial accelerations. Although the mass which is continually added to the moving shell causes no change in the tangential component of momentum, it does decrease the tangential velocity, thus further mitigating the effect of neglecting the tangential velocity.

In view of the adiabatic stage of the compression which follows the snowplow stage, the only end results required from the snowplow are: (a) the total energy of the plasma, (b) the coil currents, and (c) the plasma contour. Furthermore, since the plasma contour at the snowplow end is significant only as it affects the coil currents which, in turn, are largely governed by the self-inductance of the circuits, items (b) and (c) are of secondary importance. Thus, the approximations of the snowplow sequence are serious only to the extent that they affect the calculation of the total work done on the plasma by the magnetic field up to crowbar time. These approximations limit the quantitative accuracy of the final equilibrium position, but probably do not affect the qualitative result that a plasma equilibrium exists for the conditions of the compression which this computer sequence simulates. This conclusion is supported by the fact that the equilibrium position can be altered in a controlled and predictable way by changing some of the parameters of the compression, e.g., the initial voltages on the capacitors.

\section{ADIABATIC COMPRESSION}

During the adiabatic compression stage of the computer sequence, the internal gas pressure (assumed uniform) is subtracted from the computed magnetic pressure for each boundary segment and the movement of that boundary segment is taken to be proportional to the pressure difference. The physically complicated pro- cess of the imploding plasma colliding with itself at the center of the vessel is ignored and instead, the previously ordered particle velocities are assumed to become immediately randomized by collisions and a thermodynamic equilibrium state approached. A more elaborate and sophisticated calculation incorporating the complexities of magnethydrodynamic theory is not in the spirit of this physically oriented phenomenological calculation. Consequently, the simple proportionality relationship $\Delta x_{j}$ $=\alpha_{j}\left[P_{i}(\mathrm{mag})-P\right.$ (gas) $]$ seems an appropriate model to represent the gradual approach to a constant pressure surface, which constitutes equilibrium. Note that $\alpha_{j}$ may be chosen to be different for each plasma section. In a refinement of this adiabatic approach to equilibrium, the magnetic pressure acting on each section of the plasma is compared with $P$ (gas). If the approach toward $P$ (gas) for that iteration was an overshoot for a given plasma section, then $\alpha_{j}$ for that section was diminished (usually by 0.5 ) for the next iteration. If that approach was an undershoot, then $\alpha_{j}$ was increased (usually by a factor of 1.1). This refinement was an attempt to take into account the differing slopes in the effective potential well for different parts of the plasma surface.

The gas pressure is changed after each movement in such a way as to keep $P V^{\gamma}=$ const as for the adiabatic compression of an ideal gas. This adiabatic compression portion of the computer sequence continues until the current in either the $\theta$ coil, the I coil, or the $C$ coil begins to decrease. At that time, the "crowbar" condition is introduced into the computer sequence.

It should be emphasized that the adiabatic compression portion of the computer sequence does not model an actual dynamical process, i. e., no equation of motion is solved here. What is carried out rather is a search for an equilibrium state which is consistent with the plasma internal energy at the end of the snowplow, consistent also with the increasing currents in the coils due to the still-discharging capacitors, and consistent with the flux conditions on the various conductors. This adiabatic sequence could be carried out in a single step if the coil currents at crowbar time were known. The process used allows for coarse modeling of the changing coil inductance due to its coupling with the changing plasma and thus may give a more valid equilibrium configuration at crowbar time simply because the coil currents are perhaps determined more accurately than if some other method were used to estimate them.

\section{CROWBAR CONDITION}

In the crowbar condition portion of the computer sequence, the flux condition for the $\theta$ coil (changed from that used during the snowplow compression and the adiabatic compression stages of the sequence) is

$$
\dot{\Phi}_{\theta}=-L_{\theta} \ddot{Q}_{1}-R \dot{Q}_{1} \text {. }
$$

Note that a circuit resistance has been introduced here. The differential equation governing the current in the $\theta$ coil becomes, where the notation is that of Sec. II

$$
\ddot{Q}_{1}+\beta^{\prime} \dot{Q}_{1}=0 \text {, }
$$

where 


$$
\beta^{\prime}=\left(L_{e}+\frac{\mu_{0}}{2 \pi} \int_{s_{\theta}} \frac{d s}{r}-\frac{\mu_{0}}{2 \pi} \int_{s_{p}} \frac{d s}{r}\right)^{-1}\left(R-\frac{\mu}{2 \pi} \int_{c_{p}} \frac{v_{p}}{r} d l\right) .
$$

Solving this equation for the $\theta$ coil current $\dot{Q}_{1}$, one obtains

$$
\dot{Q}_{1}(t)=\dot{Q}_{1}\left(t_{0}\right) \exp \left[-\beta^{\prime}\left(t-t_{0}\right)\right],
$$

where the integration constant has been evaluated in terms of the current at $t=t_{0}$. Once more, the nonconstancy of $\beta^{\prime}$ is taken into account by the technique of short time iterations for each of which a new value of $\beta^{\prime}$ is introduced.

The flux conditions for the $\mathrm{I}$ and $\mathrm{C}$ coils also change in the crowbar portion of the computer sequence. They become

$$
\begin{aligned}
& \dot{\Phi}_{I}=-L_{I} \ddot{Q}_{I}-R_{I} \dot{Q}_{I}, \\
& \dot{\Phi}_{C}=-L_{C} \ddot{Q}_{C}-R_{C} \dot{Q}_{C},
\end{aligned}
$$

where $R_{I}$ and $R_{C}$ are the resistances of the crowbarred $I$ and $C$ coil circuits respectively. Making use of

$$
\dot{Q}_{I}=\sum_{i=1}^{n_{1}} J_{1}^{B}
$$

and

$$
\dot{Q}_{C}=\sum_{i=1_{1}^{+1}}^{n_{1}+n_{2}} J_{i}^{Z}
$$

and the notation of Sec. II one may write the flux condition equations in matrix form

$$
\mathrm{L}^{E}+\mathbf{R J}^{E}+\frac{d}{d t}\left(\mathbf{K J ^ { E }}\right)=0,
$$

where the matrices $\mathbf{L}$ and $\mathbf{R}$ are both block diagonal having $L_{I}$ and $R_{l}$, respectively, as all the elements of the first $n_{1} \times n_{1}$ block and $L_{c}$ and $R_{c}$, respectively, as all the elements of the next $n_{2} \times n_{2}$ block. Rearranging this equation, one gets

$$
\dot{\mathrm{J}}^{E}+(\mathrm{L}+\mathrm{K})^{-1}(\mathbf{R}+\dot{\mathbf{K}}) \mathrm{J}^{E}=0,
$$

which has the solution

$$
\mathrm{J}^{E}(t)=\exp \left[-(\mathrm{L}+\mathrm{K})^{-1}(\mathbf{R}+\dot{\mathrm{K}})\left(t-t_{0}\right)\right] \mathrm{J}^{E}\left(t_{0}\right)
$$

\begin{tabular}{|c|c|c|c|c|c|c|c|c|c|c|c|}
\hline \multirow{2}{*}{$\frac{\text { Coil }}{\theta}$} & \multirow{2}{*}{$\begin{array}{l}\text { Initial } \\
\text { voltage } \\
\text { (V) }\end{array}$} & \multirow{2}{*}{$\begin{array}{l}\text { Vol tage at } \\
\text { crowbar time } \\
\text { (V) }\end{array}$} & \multicolumn{2}{|c|}{$\begin{array}{l}\text { Current at } \\
\text { crowbar time } \\
\text { (A) }\end{array}$} & \multirow{2}{*}{$\begin{array}{l}\begin{array}{l}\text { Self- } \\
\text { inductance } \\
(\mathrm{nH})\end{array} \\
33\end{array}$} & \multicolumn{2}{|c|}{$\begin{array}{l}\text { Mutual } \\
\text { inductance } \\
\text { with plasma at } \\
\text { crowbar time } \\
\text { (nH) }\end{array}$} & \multirow{2}{*}{$\begin{array}{l}\text { External lead } \\
\text { inductance } \\
\text { (nH) }\end{array}$} & \multirow{2}{*}{$\begin{array}{l}\text { Resistance } \\
\text { after } \\
\text { crowbarring } \\
(\Omega)\end{array}$} & \multirow{2}{*}{$\begin{array}{l}\text { Capacitance } \\
(\mu \mathrm{F}) \\
16.7\end{array}$} & \multirow{2}{*}{$\begin{array}{l}\begin{array}{l}\text { Energy } \\
\text { (J) }\end{array} \\
20900\end{array}$} \\
\hline & & & -1 & $1 \times 10^{6}$ & & 1.6 & & & & & \\
\hline I & 30000 & -4400 & +9 . & $7 \times 10^{4}$ & 330 & & & 30.0 & $3.58 \times 10^{-5}$ & 1.858 & 836 \\
\hline C & -40000 & 11000 & -1 & $3 \times 10^{5}$ & 230 & & & 30.0 & $1.9 \times 10^{-5}$ & 3.716 & 3000 \\
\hline \multicolumn{3}{|c|}{ Major radius of torus } & \multicolumn{3}{|c|}{$0.25 \mathrm{~m}$} & & \multicolumn{3}{|c|}{$\begin{array}{l}\text { Total energy transfer to plasma } \\
\text { by crowbar time }\end{array}$} & \multicolumn{2}{|l|}{$1500 \mathrm{~J}$} \\
\hline \multicolumn{4}{|c|}{ Half-height of plasma at crowbar time } & \multicolumn{2}{|l|}{$0.027 \mathrm{~m}$} & & \multicolumn{3}{|c|}{ Preionization temperature } & \multicolumn{2}{|c|}{$1000^{\circ} \mathrm{K}(0.09 \mathrm{eV})$} \\
\hline \multicolumn{4}{|c|}{$\begin{array}{l}\text { Initial volume of plasma (volume } \\
\text { of vacuum chamber) }\end{array}$} & \multicolumn{2}{|l|}{$0.03 \mathrm{~m}^{3}$} & & \multicolumn{3}{|c|}{$\begin{array}{l}\text { Equivalent temperature of plasma } \\
\text { at crowbar time }\left(n k T=\frac{2}{3} E\right)\end{array}$} & \multicolumn{2}{|c|}{$3.19 \times 10^{6}{ }^{\circ} \mathrm{K}(275 \mathrm{eV})$} \\
\hline \multicolumn{3}{|c|}{$\begin{array}{l}\text { Volume of plasma at crowbar } \\
\text { time }\end{array}$} & \multicolumn{3}{|c|}{$0.0036 \mathrm{~m}^{3}$} & & \multicolumn{3}{|c|}{$\begin{array}{l}\text { Total number of particles in plasma } \\
\text { Mass density for hydrogen initially }\end{array}$} & $\begin{array}{l}3.0 \times 10^{19} \\
1.67 \times 10^{-6} k\end{array}$ & $\mathrm{~kg} / \mathrm{m}^{3}$ \\
\hline \multirow{2}{*}{\multicolumn{3}{|c|}{$\begin{array}{l}\text { Compression ratio (initial plasma } \\
\text { volume/plasma volume } \\
\text { @crowbar time) }\end{array}$}} & \multirow{2}{*}{\multicolumn{3}{|c|}{8.3}} & & \multicolumn{3}{|c|}{$\begin{array}{l}\text { Mass density at crowbar time for } \\
\text { hydrogen }\end{array}$} & \multicolumn{2}{|c|}{$1.39 \times 10^{-5} \mathrm{~kg} / \mathrm{m}^{3}$} \\
\hline & & & & & & & \multicolumn{3}{|c|}{ Particle density initially } & \multicolumn{2}{|l|}{$10^{21} / \mathrm{m}^{3}$} \\
\hline \multicolumn{4}{|c|}{$\begin{array}{l}\text { Aspect ratio (major radius of torus/ } \\
\text { half-height of plasma } \\
\text { @crowbar time) }\end{array}$} & 9.3 & & & $\begin{array}{l}\text { Partic } \\
\text { Time }\end{array}$ & $\begin{array}{l}\text { le density at } \mathrm{cr} \\
\text { increment befor }\end{array}$ & $\begin{array}{l}\text { owbar time } \\
\text { ce crowbarring }\end{array}$ & $\begin{array}{l}8.3 \times 10^{21} / \mathrm{m} \\
5 \mathrm{nsec}\end{array}$ & \\
\hline Fillin & gas pres & & & $4.14 \mathrm{nt} / \mathrm{m}$ & $a^{2}(0.031$ Tor & & Time & increment after & crowbarring & $1.0 \mu \mathrm{sec}$ & \\
\hline $\begin{array}{l}\text { Gas p } \\
\quad \text { (to }\end{array}$ & $\begin{array}{l}\text { essure af } \\
\left(000^{\circ} \mathrm{K}\right)\end{array}$ & preionization & & $13.81 \mathrm{nt} /$ & $\mathrm{m}^{2}(0.104 \mathrm{Tc}$ & orr) & Time & at snowplow end & & $0.3 \mu \mathrm{sec}$ & \\
\hline Gas $p$ & essure at & owbar time & & $2.80 \times 10^{5}$ & $\mathrm{nt} / \mathrm{m}^{2}(2109$ & Torr) & Time & at crowbarring & of circuits & $1.05 \mu \mathrm{sec}$ & \\
\hline $\begin{array}{l}\text { Magn } \\
\text { surf }\end{array}$ & $\begin{array}{l}\text { tic field } s \\
\text { tee at crov }\end{array}$ & $\begin{array}{l}\text { ugth on plasma } \\
\text { ar time }\end{array}$ & & 0.83 Tesl & & & Iterat & ion number at $\mathrm{st}$ & nowplow end & 68 & \\
\hline Total & energy of & pacitor banks & & $24710 \mathrm{~J}$ & & & Iterati & on number at $\mathrm{cr}$ & rowbar time & 210 & \\
\hline
\end{tabular}

where the matrix exponential is to be understood in terms of a power series expression. The plasma currents, $\mathrm{J}^{P}$, are obtained in the usual way from

$$
\mathrm{J}^{P}=-\left(\mathbf{L}^{P P}\right)^{-1} \mathrm{~L}^{P E} \mathrm{~J}^{E} .
$$

After the currents $\mathrm{J}^{E}$ and $\mathrm{J}^{P}$ are found in this way, the magnetic pressure on the plasma surface is computed in the same way as for the adiabatic compression

TABLE I. Parameters for the computer sequence illustration. 


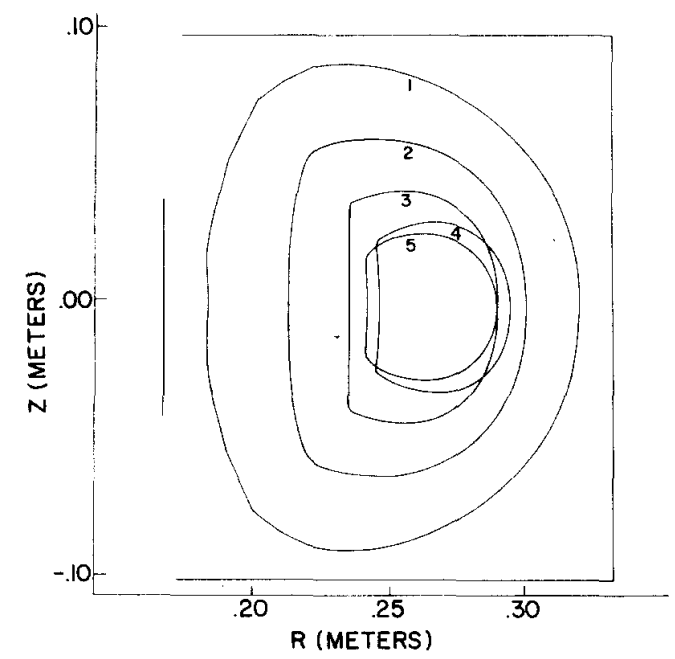

FIG. 5. The plasma surface position in the poloidal plane of a topolotron compression. Positions shown are for (1) 0.01 , (2) 0.32 , (3) 0.76, (4) $1.05 \mu$ sec after the compression was initiated, and (5) the equilibrium position of the plasma. Contour (2) is near the end of the snowplow phase and contour (4) is near the crowbar time.

stage of the computer sequence. Moreover, the movement of the plasma toward a constant pressure surface is also carried out by the same procedure as during the adiabatic compression.

\section{RESULTS OF THE APPLICATION OF THE COMPUTER SEQUENCE TO THE TOPOLOTRON}

The computer sequence discussed here was used as an aid in the selection of coil and energy bank parameters for a topolotron. That computer sequence represents a practical way of taking into account the intimate coupling between the conducting plasma under the influence of the electromagnetic forces present. Several sets of parameters were used in successive runs of the computer sequence. Such a parameter set which gives promise for a viable physical apparatus is listed in Table I. The results of the computer-simulated compression are shown in graphical form as Figs. 5-8. In Fig. 5 , the plasma surface position in the poloidal plane is shown for successive stages of the compression. In Fig. 6, the position of the $I$ and $C$ coils and the plasma surface are shown. Also drawn are several constant magnetic flux function $(\psi)$ lines. The difference in $\psi$ be-

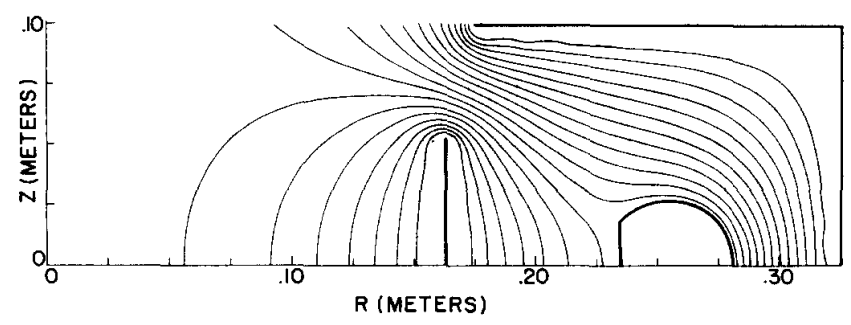

FIG. 6. A computer-drawn graph of the poloidal magnetic field lines in the neighborhood of a compressed high beta plasma. The positions of the $I$ and $C$ coils and plasma surface are shown for an equilibrium configuration of the plasma.

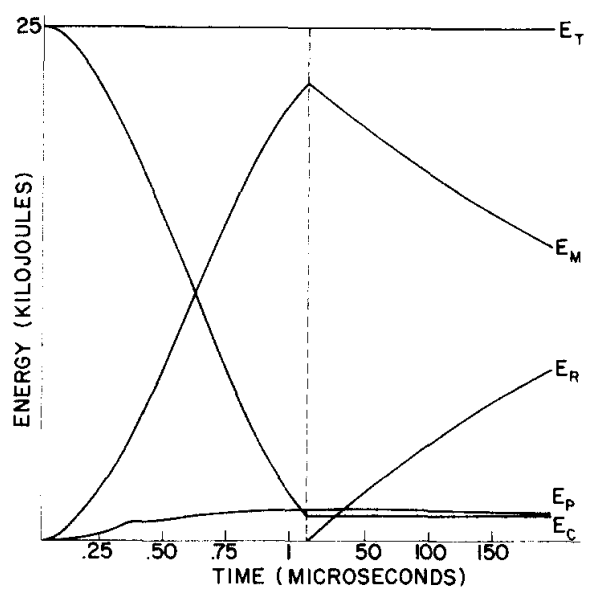

FIG. 7. A graph showing the energy in $\left(E_{c}\right)$ the capacitors, $\left(E_{m}\right)$ the magnetic field, $\left(E_{p}\right)$ the plasma, $\left(E_{R}\right)$ dissipated in the resistance and $\left(E_{T}\right)$ the total energy, as a function of time during the simulated plasma compression. Note the change in the time scale after crowbarring indicated by the dotted line.

tween pairs of adjacent lines is a constant in a given plot so that the graph is the usual magnetic field line plot for the poloidal component of magnetic field. The graph shown is the equilibrium configuration. The following features are evident from the plots and from the data of Table I. Except for the first few iterations of the computer sequence, the discrete surface segment approximation used gives a good approximation to a continuous conductor both for the plasma surface and for the external coils. This fact is evident from the lack of significant penetration of the magnetic field lines (constant $\theta$ lines) into the conductor and from the smoothness of the $\psi$ lines even near the conducting surfaces. The shape of the plasma surface does not change drastically during the snowplow portion of the compression. ${ }^{10}$ The shapes of the poloidal cross section of the $\theta, \mathbf{I}$, and $\mathrm{C}$ coils do not seem to affect this result. The $I$ and $C$ coils do affect the equilibrium shape

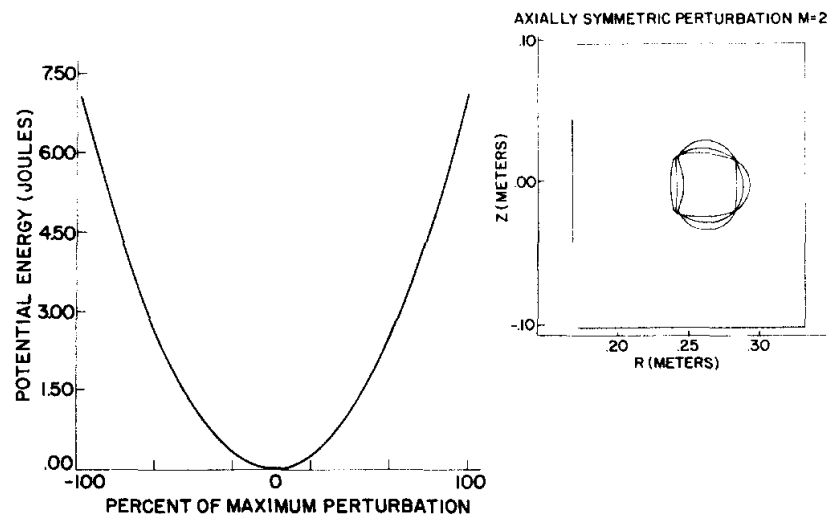

FIG. 8. A graph showing the work done by the net force (excluding the perturbing force) acting on the plasma surface during an axially symmetric perturbation. The work is interpreted as a potential energy and is plotted to show the minimum at equilibrium. The $M=2$ perturbation is shown together with its potential well: The abscissa shows percent of the maximum perturbation. 
of the plasma in a significant way; it is the influence of these coils that produces the limit cycle (asymptotic) form of the magnetic field configuration on the plasma surface. The end of the snowplow portion of the sequence occurred at $0.3 \mu \mathrm{sec}$ after the capacitor discharge began, which is 0.07 of a period. This time is is that for which the work done on the plasma (compression heating energy), when randomized, would give rise to an (ideal) internal gas pressure equal to the average magnetic pressure acting on the plasma surface. The magnetic pressure on the surface of the plasma is not uniform at the end of the snowplow sequence, of course, since the plasma is not in equilibrium then. Equilibrium is approached during the adiabatic compression sequence, however (in this computer model). The circuits are simultaneously crowbarred at $1.05 \mu$ sec after the capacitor discharge was initiated.

The volume of the plasma decreased by a factor of 0.19 during the snowplow portion of the computer sequence. A further decrease in volume occurs during the adiabatic compression phase, making the final volume a factor of one tenth of the initial volume. This decrease comes almost entirely from the decrease in the cross-sectional area of the toroidal plasma; the major radius remains essentially unchanged during the compression. The final aspect ratio (i.e., at crowbarring) is 9. 3, based on the definition: $R=$ major radius/half-height of plasma. The limit cycle is clearly visible in the graph (Fig. 6) as the position of minimum radius (one limit cycle in the half-plane).

The graphs of Figs. 5 and 6 show the existence, by construction, of a simulated plasma equilibrium for the given coil geometry and the parameters listed in Table I. The magnetic field on the plasma surface has limit cycles, and therefore possesses the asymptotic magnetic field configuration which has "structural stability" in the topological language discussed in the introduction. If a perturbing force displaces the plasma in such a way that its perfect conductor character is retained and such that the perturbation is axially symmetric, then the computer sequence shows that the same equilibrium position will be regained by the plasma when the perturbing force is removed. This return occurs under steady state conditions where the circuits have been crowbarred and the decay of the currents is much slower than the perturbation. The restriction to axially symmetric perturbations is purely a computational restriction and no information regarding asymmetric perturbations may be inferred.

The energy involved in the plasma compression can be easily calculated using the parameters of the computer sequence. This energy calculation has provided substantiation of energy conservation during the three stages of the computer sequence, and it has proved to be a convenient debugging tool. Figure 7 shows the energies in the capacitors, the magnetic field, the plas$\mathrm{ma}$, and the energy dissipated in the resistance after crowbarring, as well as the total of these energies for the example discussed above. The work done by the net force (excluding any perturbing force) acting on the plasma surface can also be readily calculated. This work, when interpreted as potential energy is a minimum for the equilibrium configuration of the plasma; that is, the plasma at equilibrium resides in a potential energy well, at least for perturbations which are axially symmetric. The potential well is dependent upon the presence of the external I, C, and $\theta$ coils, and it is a manifestation, in part, of the so-called "wall stabilization." Figure 8 shows this potential energy well for the perturbation sketched on the graph.

The foregoing discussion has been directed toward the application of the computer sequence to the topolotron. It has been pointed out above, however, that an application of the sequence may be made to any axially symmetric toroidal device with orthogonal conductors in the sharp boundary approximation. Within the limitations caused by its simplicity, this sequence provides confidence that a conjectured plasma equilibrium exists as the end product of an actual magnetic compression. It also reveals design parameters of an apparatus and gives insight into the range of variation which should be provided in those parameters to insure that the device can be adjusted or tuned to compensate for approximations in the computer model.

\section{ACKNOWLEDGMENTS}

The authors wish to acknowledge valuable discussions with A. L. Gardner, who suggested the method, and with J. H. Gardner, L. V. Knight, and H. R. P. Ferguson.

${ }^{2}$ J. H. Gardner, R. W. Bass, L. Fearnley, H. R. P. Ferguson, B. K. Harrison, K. M. Larsen, and H. M. Nelson, Proc. Utah Acad. Sci., Arts, Lett. 50, 1 (1973); R. W. Bass, Bull. Am. Phys. Soc. 19, 657 (1974).

${ }^{2}$ H. M. Nelson, R. W. Bass, L. Fearnley, H. R. P. Ferguson, J. H. Gardner, B. K. Harrison, K. M. Larsen, D. E. Nielson, G. Cook, L. Scott, R. Wilson, and W. Young, Proc. Utah Acad. Sci., Arts, Lett. 50, 47 (1973).

${ }^{3}$ K. F. McKenna, R. Kistual, and K. S. Thomas, Phys. Rev. Lett. 32, 409 (1974).

${ }^{4}$ H. Alfven, in Cosmic Plasma Physics (Plenum, New York, 1972), p. 1 .

${ }^{5}$ F. L. Ribe, Los Alamos Scientific Laborator $J^{*}$ Report LAMS 2471 (1960) and S. Itoh, N. Fujisawa, and K. Yamamoto, Phys. Fluids 12, 220 (1969).

${ }^{6} \mathrm{~J}$. Berkowitz, H. Grad, and H. Rubin, in Proceedings of the $U$. N. Second International Conference on the Peaceful Uses of Atomic Energy (United Nations, Geneva, 1958), Vol. 31, p. 177.

${ }^{7}$ D. C. Stevens, Phys. Fluids 17, 222 (1974).

${ }^{8} \mathrm{~J}$. A. Stratton, Electromagnetic Theory (McGraw-Hill, New York, 1941), pp. 263, 264.

${ }^{9} \mathrm{Z}$. W. Grover, Inductance Calculations (Van Nostrand, New York, 1946).

${ }^{10}$ I. F. Kuartskjava, K. N. Kervakidze, and Y. S. Gvaladze, Zh. Tekh. Fiz. 30, 1321 (1960) [Sov. Phys.-Tech. Phys. 5, 1253 (1961). 